\title{
Study of the tolerance of infliximab infusions with or without betamethasone premedication in patients with active rheumatoid arthritis
}

\author{
J Sany, M J Kaiser, C Jorgensen, G Trape
}

Ann Rheum Dis 2005;64:1647-1649. doi: 10.1136/ard.2005.037499

\begin{abstract}
Objectives: To assess the incidence of infusion reactions in patients with rheumatoid arthritis (RA) receiving infliximab treatment with and without betamethasone premedication. To determine whether patients with an atopic diathesis had a better response to corticosteroid pretreatment than those without, and the course of patients' functional capacity and quality of life.

Patients and methods: A prospective, multicentre, randomised, double blind phase 4 study of 355 patients with RA in two groups: group A received betamethasone and group $B$ placebo, before a 36 week infusion treatment with infliximab. Incidence and severity of infusion reactions from infliximab treatment were assessed.

Results: The incidence of reactions to infliximab infusion was $<5 \%$. More infusion reactions occurred with betamethasone pretreatment than with placebo. Response to infliximab of patients with atopic backgrounds did not differ in the presence or absence of betamethasone from that of nonatopic patients. Mean Health Assessment Questionnaire score improved by $47 \%$ at week 24 , quality of life assessed by Short Form-36 improved in mental and physical component subscales.

Conclusions: Incidence of infusion reactions with infliximab was low and their severity generally mild, but betamethasone pretreatment did not decrease the incidence and severity of infusion reactions. Betamethasone, therefore, is not recommended as a systematic prophylactic measure, even in atopic patients.
\end{abstract}

\footnotetext{
A common feature of biological treatment, including anti-tumour necrosis factor $\alpha(\mathrm{TNF} \alpha)$ treatment with available drugs in America and Europe, is an immune response to the administered drug. Infusion reactions with infliximab occur in about $5 \%$ of infusions, ${ }^{12}$ and most reactions resolve when the infusion rate is decreased or treatment with acetaminophen, antihistamines, steroids, and/or epinephrine is given. ${ }^{1}$ Patients who have had infusion reactions are often pretreated with corticosteroids ${ }^{1-3}$ to decrease proactively the incidence and severity of subsequent infusion reactions.

This study aimed at comparing the incidence and severity (minimum, mild, moderate, severe) of infusion reactions resulting from standard infliximab administration in the presence or absence of corticosteroid pretreatment and investigating whether patients with an atopic diathesis were more likely to respond to corticosteroid pretreatment by having fewer and/or less severe infusion reactions, than nonatopic patients.
}

\section{PATIENTS AND METHODS}

\section{Patient selection}

Patients were eligible for the study if they were aged between 18 and 80 years with active rheumatoid arthritis (RA) defined by the 1987 American Rheumatism Association revised criteria ${ }^{4}$ according to the infliximab indications and were resistant to at least one disease modifying antirheumatic drug. Patients had to have been receiving a stable dose of methotrexate of $\geqslant 7.5 \mathrm{mg} /$ week for at least 1 month. Azathioprine (1.5-3 mg/kg/day) or $20 \mathrm{mg}$ leflunomide could be substituted in patients intolerant to methotrexate. Any other disease modifying antirheumatic drugs were discontinued for at least 4 weeks before the start of the trial.

\section{Study protocol}

Patients were randomly assigned to receive either a standard 2 hour intravenous infusion of infliximab at $3 \mathrm{mg} / \mathrm{kg}$ at weeks $0,2,6,14$, and 22, with betamethasone (Celestene) at $0.15 \mathrm{mg} / \mathrm{kg} 30$ minutes before each infliximab infusion (group A), or the same dose of infliximab preceded by an intravenous placebo (group B). Infusion reactions were defined as the occurrence of chills, pruritus, urticaria, chest pain, difficulty breathing, hypotension, hypertension, dyspnoea, and/or tachycardia during the course of, or within, 2 hours after the infliximab infusion. The percentage and severity of the delayed reactions were calculated in the two patient groups and compared, based on a form completed by the patient at the time of each clinical symptom suggesting a delayed reaction. Atopic diathesis was assessed by a questionnaire, including childhood rhinitis, conjunctivitis, and alimentary allergies. Additional assessments included the course of the patients' functional capacity evaluated by the Health Assessment Questionnaire (HAQ) Disability Index ${ }^{5}$ and quality of life evaluated by the Short Form-36 (SF-36) questionnaire.

\section{Statistical analyses}

The baseline characteristics of the two groups were compared on an intention to treat basis using Student's $t$ test. Qualitative variables were compared using the $\chi^{2}$ test or Fisher's exact test. For patients having several immediate reactions, the one that was most severe was used for analysis.

\section{RESULTS}

Between October 2001 and May 2002, 365 patients were included in the study, 355 of whom were available for analysis in group A (betamethasone, $\mathrm{n}=179$ ) or B (placebo, $n=176$ ). Table 1 shows that the two groups were evenly balanced, except that group A had slightly fewer women than group B.

Abbreviations: $\mathrm{HAQ}$, Health Assessment Questionnaire; RA, rheumatoid arthritis; SF-36, Short Form-36 


\begin{tabular}{|c|c|c|c|}
\hline Characteristic & $\begin{array}{l}\text { Group A } \\
\text { Infliximab + } \\
\text { placebo } \\
(n=176)\end{array}$ & $\begin{array}{l}\text { Group B } \\
\text { Infliximab + } \\
\text { betamethasone } \\
(n=179)\end{array}$ & p Value \\
\hline Women (\%) & 71 & 80 & 0.05 \\
\hline Mean age (years) & 52.3 & 53.2 & 0.47 \\
\hline Mean weight $(\mathrm{kg})$ & 65.5 & 68.3 & 0.08 \\
\hline Mean number of swollen joints & 10.6 & 10.7 & 0.89 \\
\hline Mean number of tender joints & 14.5 & 14.8 & 0.68 \\
\hline Mean morning stiffness (min) & 116.5 & 118.9 & 0.81 \\
\hline Mean ESR $(\mathrm{mm} / 1 \mathrm{st} h)$ & 34.0 & 35.4 & 0.60 \\
\hline Mean CRP (mg/l) & 35.4 & 30.5 & 0.17 \\
\hline Mean disease duration (years) & 10.9 & 11.4 & 0.58 \\
\hline Patients with surgical interventions (\%) & 43.2 & 49.7 & 0.22 \\
\hline Mean number of interventions & 3.2 & 2.9 & \\
\hline Mean number of DMARDs for RA & 3.9 & 3.9 & 0.86 \\
\hline \multicolumn{4}{|l|}{ Associated drugs (\%) } \\
\hline Methotrexate & 81.2 & 85 & 0.36 \\
\hline Azathioprine & 2.3 & 1.6 & \\
\hline Leflunomide & 16.5 & 13.4 & \\
\hline Oral corticosteroids & 84.7 & 77.1 & 0.07 \\
\hline Presence of one atopy (\%) & 23.9 & 22.9 & 0.83 \\
\hline Eczema (\%) & 10.8 & 14 & 0.40 \\
\hline Hay fever (\%) & 11.4 & 8.9 & 0.45 \\
\hline Rhinitis or allergic rhinitis or conjunctivitis (\%) & 2.3 & 4.5 & 0.25 \\
\hline Food allergy (\%) & 3.4 & 5.0 & 0.45 \\
\hline Confirmed asthma (\%) & 6.3 & 4.5 & 0.46 \\
\hline
\end{tabular}

\section{Clinical response}

The overall incidence of infusion reactions for both groups combined was $3.8 \%$. Of the 840 infliximab infusions in the betamethasone group, $42(5.0 \%)$ resulted in infusion reactions, twice the number of the placebo group, which had 21 $(2.5 \%)$ infusion reactions out of 827 infusions (fig 1A). In the group of patients who were treated with betamethasone, 30/ $179(16.8 \%)$ patients had an infusion reaction compared with $18 / 176(10.2 \%)$ patients in the control group (fig 1B). The presence or absence of an atopic background had no apparent effect on the incidence of infusion reactions: of the patients who reported an atopic diathesis, $10.8 \%$ had infusion reactions compared with $14.3 \%$ in the group reporting no history of atopy (fig 2A). The use of oral steroids during the study did not affect the incidence of infusion reactions (fig 2 B), and there was no difference between men and women in the number of infusion reactions in each treatment group (data not shown).

Infliximab was routinely administered in an induction phase at 0,2 , and 6 weeks, followed by a maintenance phase every 8 weeks thereafter. The number of infusion reactions was similar in the placebo and betamethasone groups during both induction and maintenance phases $(p=0.61)$. The overall intensity of infusion reactions was minimum to
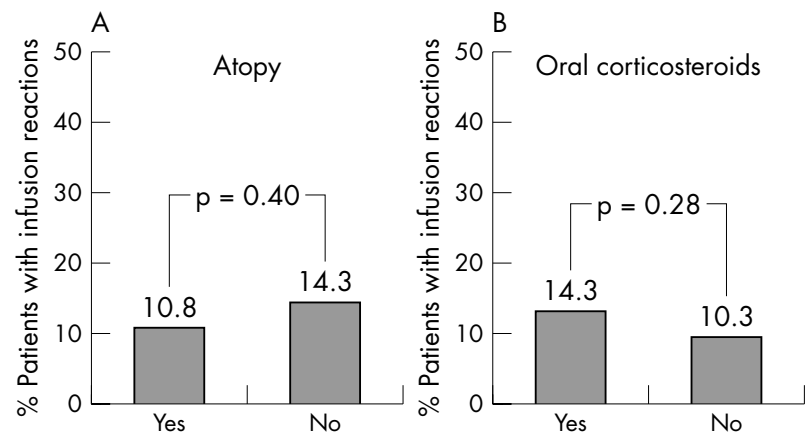

Figure 2 Percentage of patients in whom infusion reactions occurred in the presence or absence of $(A)$ an atopic background and $(B)$ the use of oral corticosteroids.

moderate and most infusion reactions resolved with treatment after infusion, or spontaneously. Betamethasone pretreatment did not ameliorate the severity of infusion reactions in comparison with the placebo group. No delayed hypersensitivity reactions were recorded.

Mean HAQ scores in this study improved by 0.47 at week 24. These scores and their improvements were similar in both

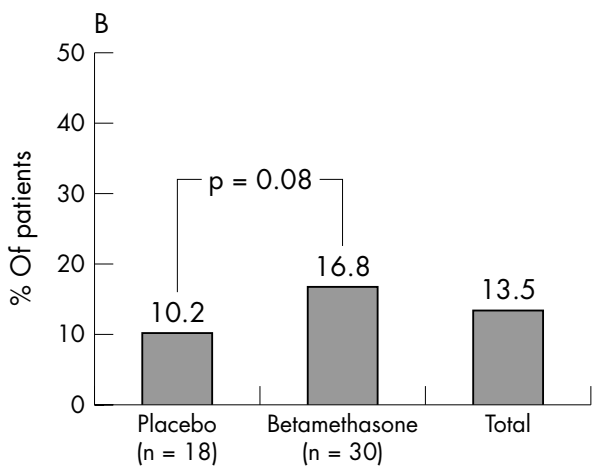

Figure 1 (A) Percentage of infusions in which infusion reactions occurred. (B) Percentage of patients in whom infusion reactions occurred. 
placebo and betamethasone treatment groups during both the induction and maintenance phase of treatment. SF-36 scores also improved over the course of the study for all measures, and no difference due to betamethasone treatment was found for any of the individual assessments.

\section{DISCUSSION}

As far as we know, this study is the only infliximab study to examine the usefulness of a corticosteroid prescribed specifically to reduce the incidence and severity of infusion reactions. Seventy seven per cent of the reactions were low to moderate in intensity and $97 \%$ of the reactions resolved spontaneously or with standard treatment of symptoms.

Prophylactic betamethasone did not ameliorate the incidence or intensity of the infusion reactions recorded. No added benefit of betamethasone pretreatment was found in any patient subgroups in this study, including patients with an atopic diathesis. This is consistent with the concept that these infusion reactions may not be IgE mediated acute hypersensitivity events. ${ }^{1-6}$

Interestingly, in this study the incidence of infusion reactions during the first infusion was $3.4 \%(6 / 76)$ in the placebo group and 5.0\% (9/79) in the corticosteroid group; a higher incidence than previously reported. The occurrence of infusion reactions during the first infusion supports the hypothesis that at the first infusion, reactions are not always related to the presence of antibodies to infliximab.

It has been well established that the concomitant administration of immunosuppressant drug treatment can help to decrease infusion reactions in patients with RA resulting from anti-tumour necrosis factor $\alpha$ treatment with infliximab. ${ }^{3}$ However, based on the results of this study, the systematic proactive use of betamethasone is not recommended to improve the management of infusion reactions. Rather, careful monitoring of each patient individually remains the optimal approach.

\section{ACKNOWLEDGEMENTS}

We thank all the French rheumatologists who took part in this study.

\section{Authors' affiliations}

J Sany, M J Kaiser, C Jorgensen, Service d'Immuno-Rhumatologie, Hôpital Lapeyronie, 371 avenue du Doyen Gaston Giraud, 34295 Montpellier Cedex 5, France

G Trape, Laboratoire Schering-Plough, 92 rue Baudin, 92307 Levallois Perret, France

Correspondence to: Dr J Sany, j-sany@chu-montpellier.fr

Accepted 13 April 2005

\section{REFERENCES}

1 Cheifetz A, Smedley M, Martin S, Reiter M, Leone G, Mayer L, et al. The incidence and management of infusion reactions to infliximab: a large center experience. Am J Gastroenterol 2003;98:1315-24.

2 Schaible TF. Long term safety of infliximab. Can J Gastroenterol 2000;14(suppl C):29-32C

3 Sandborn WJ. Preventing antibodies to infliximab in patients with Crohn's disease: optimize not immunize. Gastroenterology 2003; 124:1140-5.

4 Arnett FC, Edworthy SM, Bloch DA, McShane DJ, Fries JF, Cooper NS, et al. The American Rheumatism Association 1987 revised criteria for the classification of rheumatoid arthritis. Arthritis Rheum 1988;31:315-24.

5 Fries JF, Spitz P, Kraines RG, Holman HR. Measurement of patient outcome in arthritis. Arthritis Rheum 1980;23:137-45.

6 Farrell RJ, Alsahli M, Jeen YT, Falchuk KR, Peppercorn MA, Michetti P. Intravenous hydrocortisone premedication reduces antibodies to infliximab in Crohn's disease: a randomized controlled trial. Gastroenterology 2003; 124:917-24. 